\title{
Effect of Isoflavones and Genistein on Glucose Metabolism in Peri- and Post-Menopausal Women: An Overview of Meta-Analysis
}

Afiat Maliehe ${ }^{1}$, Sara Ghahremani ${ }^{2}$, Sara Kharghani ${ }^{3}$, Masumeh Ghazanfarpour ${ }^{4}$, Khatereh Shariati ${ }^{5}$, Maryam Kazemi ${ }^{5}$, Talat Khadivzadeh ${ }^{5}$

'Women's Health Research Center, Department of Obstetrics and Gynecology, Faculty of Medicine, Mashhad University of Medical Sciences, Mashhad, Iran, ${ }^{2}$ Department of Pediatrics, Faculty of Medicine, Mashhad University of Medical Sciences, Mashhad, Iran, ${ }^{3}$ Department of Anesthesiology, Mashhad University of Medical Sciences, Mashhad, Iran, ${ }^{4}$ Department of Midwifery, Razi School of Nursing and Midwifery, Kerman University of Medical Sciences, Kerman, Iran, ${ }^{5}$ Department of Medical Education, Mashhad University of Medical Sciences, Mashhad, Iran

The purpose of the present overview of meta-analysis is to summarize and critically assess the effect of isoflavones and genistein on glucose metabolism among the peri- and post-menopausal women. Two independent authors searched the databases of MEDLINE, Scopus and Cochrane Library for meta-analysis. Three databases were searched from inception to January 2018. Methodological quality of each meta-analysis of randomized controlled trials was evaluated using the AMSTAR (a measurement tool used to assess systematic reviews). Four meta-analyses were included to the current overview. Fasting insulin levels and homeostatic model assessment of insulin resistance (HOMA-IR) values were significantly lower in peri-menopausal and postmenopausal. Two meta-analyses showed that treatment with isoflavones could not alter fasting blood glucose. However, one meta-analysis depicted that isoflavones significantly improved blood glucose levels in non-Asian postmenopausal women. Treatment with genistein could have significant beneficial effects on fasting insulin, blood glucose and HOMA-IR in comparison to the control group. Regardless of the population, the treatment with genistein is effective in improving fasting insulin, HOMA-IR and glucose levels. Nevertheless, the high heterogeneity among studies and poor methodology of reviews made it difficult to draw a definite conclusion on the positive impacts of soy on glucose metabolism.

Key Words: Genistein, Glucose metabolism disorders, Insulins, Isoflavones, Menopause

\section{INTRODUCTION}

The post-menopausal period reportedly can be associated with some consequences such as glucose tolerance and increase insulin resistance. Approximately $20 \%$ of women in the age range of 55 to 65 years are suffering from impaired glucose tolerance and diabetes mellitus that face this group with developing cardiovascular disease (CVD) [1]. The impaired quality of life and even subsequently mortality can occur following the glucose metabolism disorders [2]. One of the most widely used therapeutic approaches in this regard is hormone replacement therapy (HRT) that has been always accompanied with determined adverse effects such as CVD, weight loss, elevated insulin-sensitivity and impaired blood lipid profiles [1,3]. Many women in treatment now decided to discontinue HRT due to a general misconception about the relationship between hormones and cancer, as well as many of the benefits of phytoestrogens advertised by trading companies relying on minimal scientific information. Hence, the increasing tendency of postmenopausal women toward com-

Received: January 3, 2018 Revised: November 25, 2018 Accepted: December 14, 2018

Address for Correspondence: Masumeh Ghazanfarpour, Department of Midwifery, Razi School of Nursing and Midwifery, Kerman University of Medical Sciences, Haft-Bagh Highway, Kerman 7616913555, Iran

Tel: 98-91-3633-0656, E-mail: Masoumeh.Ghazanfarpour@yahoo.com, ORCID: https://orcid.org/0000-0003-4639-3711 
pounds containing phytoestrogens as complementary and alternative medicine is clearly visible in this backward trend [4]. There are four main classes in various phytoestrogen compounds, including isoflavonoids, flavonoids, coumestans, and mammalian lignans [5]. The structure and chemical nature of the isoflavones are closely similar to estradiol (E2) as the phytoestrogens are able to match with estrogen receptors [4], but their effects physiologically are greatly less than E2 [5]. Previous systematic reviews and meta-analyses have found controversial results regarding the effect of isoflavones on the glucose metabolism $[1,2,6]$. Therefore, there is further need for overview of systematic reviews to draw a definite conclusion.

\section{METHODS}

\section{Search strategy}

Two authors independently searched databases of MEDLINE, Scopus, and Cochrane Library for detect meta-analysis of randomized, controlled trials on effect of isoflavones and genistein on glucose metabolism in peri- and post-menopausal women. Three databases were searched from inception to January 2018. The following keywords in English were used in search databases with no language limitations, including (insulin [Title/Abstract] OR blood glucose [Title/Abstract]) AND (isoflavone [Title/Abstract] OR genistein [Title/ Abstract]). All references in systematic review were manually searched to search additional article and avoid overlooking any related information. Same authors also reviewed title and abstract to detect relevant publications. The consensus sessions were used to deal with any discrepancy. In case of disagreement, a third investigator judged the articles.

\section{Inclusion criteria}

Meta-analysis assessing the effect of isoflavones or genistein on at least one of the parameters of insulin, blood glucose and homeostatic model assessment of insulin resistance (HOMA-IR) in peri- and post-menopausal included into the overview.

\section{Data extraction}

Two authors using a pre-designed form extracted following data: year of publication, first author, study population and menopausal status (Table 1).

\section{Assessment of methodological quality}

The same assessors appraised the methodological quality of each meta-analysis of randomized controlled trials using the AMSTAR (a measurement tool to assess reviewer), which contains 11 items developed by Oxman et al. [7] to assess the methodological quality. Individual items were categorized as three responses of "Yes", "No", "Can’t Answer" (Table 1).

\section{RESULTS}

Process of selecting review is showed in Figure 1. Table 1 showed characteristic of 4 meta-analysis include in over view. Table 2 also showed methodological quality assessment of systematic reviews using the AM-

Search strategy of the study

Relavant studies detected in the search three data bases $(n=542)$

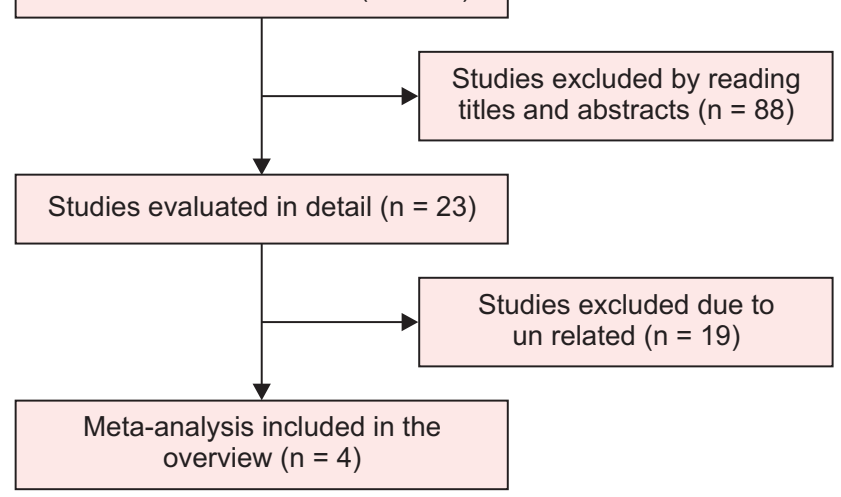

Fig. 1. Process of selecting review is showed.

Table 1. Characteristic of 4 meta-analysis included in this overview

\begin{tabular}{lcll}
\hline \multicolumn{1}{c}{ Study (year) } & Age (y) & \multicolumn{1}{c}{ Population } & \multicolumn{1}{c}{ Type intervention/control } \\
\hline Zhang et al. [1] (2013) & $52-63$ & Non-Asian postmenopausal women & Soy isoflavones supplementation/placebo \\
Liu et al. [8] (2017) & $54-60$ & Post menopause & Genistein/placebo group \\
Ricci et al. [6] (2010) & Not mentioned & $\begin{array}{c}\text { Perimenopausal and postmenopausal } \\
\text { non-Asian women }\end{array}$ & Isoflavone/placebo \\
Fang et al. [2] (2016) & $48-63$ & Menopausal women & Isoflavone/placebo \\
\hline
\end{tabular}


Table 2. Methodological quality assessment of met-analysis using the AMSTAR rating

\begin{tabular}{|c|c|c|c|c|c|c|c|c|c|c|c|}
\hline \multirow{2}{*}{ Study (year) } & \multicolumn{11}{|c|}{ AMSTAR items $^{\mathrm{a}}$} \\
\hline & 1 & 2 & 3 & 4 & 5 & 6 & 7 & 8 & 9 & 10 & 11 \\
\hline Zhang et al. [1] (2013) & Yes & Yes & Yes & Yes & $\begin{array}{l}\text { Can not } \\
\text { answer }\end{array}$ & Yes & Yes & Yes & Yes & $\begin{array}{l}\text { Can not } \\
\text { answer }\end{array}$ & No \\
\hline Liu et al. [8] (2017) & Yes & Yes & Yes & $\begin{array}{l}\text { Can not } \\
\text { answer }\end{array}$ & $\begin{array}{l}\text { Can not } \\
\text { answer }\end{array}$ & Yes & Yes & Yes & Yes & $\begin{array}{l}\text { Can not } \\
\text { answer }\end{array}$ & Yes \\
\hline Ricci et al. [6] (2010) & Yes & Not & $\begin{array}{l}\text { Can not } \\
\text { answer }\end{array}$ & Not & Not & $\begin{array}{l}\text { Can not } \\
\text { answer }\end{array}$ & Not & Not & Yes & $\begin{array}{l}\text { Can not } \\
\text { answer }\end{array}$ & Yes \\
\hline Fang et al. [2] (2016) & Yes & Not & Yes & Yes & Yes & Yes & Yes & Yes & Yes & Yes & Yes \\
\hline
\end{tabular}

AMSTAR: a meaurement tool to assess reviewer.

1: "Was an 'a priori' design provided?", 2: "Was there duplicate study selection and data extraction?", 3: "Was a comprehensive literature search performed?", 4: "Was the status of publication (i.e., grey literature) used as an inclusion criterion?", 5: "Was a list of studies (included and excluded) provided?", 6: "Were the characteristics of the included studies provided?", 7: "Was the scientific quality of the included studies assessed and documented?", 8: "Was the scientific quality of the included studies used appropriately in formulating conclusions?", 9: "Were the methods used to combine the findings of the studies appropriate?", 10: "Was the likelihood of publication bias assessed?", 11: "Was the conflict of interest stated?".

\section{STAR rating.}

\section{The effect of isoflavones on insulin}

Three meta-analyses assessed the effect of isoflavones on insulin. Ricci et al. [6] in 2010 reported that the fasting blood insulin level was significantly lower in the isoflavone compounds group (mean difference $[\mathrm{MD}]=$ $-1.39, P=0.03,95 \%$ confidence interval $[\mathrm{CI}]=-2.65$ to -0.13 , heterogeneity $\left[\mathrm{I}^{2}=61 \%\right], P=0.05 ; 4$ trials; 66 subjects for isoflavones compound and 73 subjects for placebo). Fang et al. [2] in 2016 reported that mean of insulin in soy isoflavone compounds group was significantly lower than placebo group $(\mathrm{MD}=-0.43, P=$ $0.003,95 \% \mathrm{CI}=-0.71$ to $-0.14, \mathrm{I}^{2}=76 \%, P<0.0001$; random-effect model; 12 trials; 497 women for soy isoflavone and 489 subjects for control group). Zhang et al. [1] in 2013 found that the fasting insulin levels were significantly lower in women treated with soy isoflavone supplementation compared to control group (weighted mean difference $[\mathrm{WMD}]=-0.918,95 \% \mathrm{CI}=$ -1.7 to -0.137 , heterogeneity $\left[\mathrm{I}^{2}=65 \%\right], P=0.021 ; 12$ trials; 581 subjects for soy isoflavone supplementation and 561 subjects for control group).

\section{The effect of genistein on fasting blood insulin}

Three meta-analyses assessed the effect of genistein on insulin. According to Fang et al. [2] insulin-lowering effect was more in women treated with genistein than those with placebo (MD $=-0.62, P<0.00001,95 \% \mathrm{CI}$ $=-0.82$ to $-0.41, \mathrm{I}^{2}=31 \%, P=0.201$; random-effect model; 6 trials; 341 women for treatment and 322 for control group). Ricci et al. [6] reported that the mean of fasting blood insulin was significantly lower in the genistein $(\mathrm{MD}=-1.32, P<0.00001,95 \% \mathrm{CI}=-1.78$ to -0.86 , heterogeneity $\left[\mathrm{I}^{2}=0 \%\right], P=0.48 ; 3$ trials; 236 for isoflavones and 217 for placebo) in comparison to placebo. Liu et al. [8] found that treatment with genistein can significantly reduce fasting insulin in comparison to placebo $(\mathrm{WMD}=-1.92,95 \% \mathrm{CI}=-3.04$ to -0.79 , $P=0.0008$, heterogeneity $\left[\mathrm{I}^{2}=55 \%\right], P=0.021 ; 6$ trials; 306 subjects for genistein and 285 subjects for control group).

\section{Fasting blood glucose}

\section{The effect of isoflavone on fasting blood glucose}

Three meta-analyses assessed the effect of isoflavone on fasting blood glucose. In Ricci et al's meta-analysis in 2010 [6], the mean of fasting blood glucose was not different between soy isoflavone group and placebo group $(\mathrm{MD}=-2.16,95 \% \mathrm{CI}=-5.21$ to $3.35, P=0.17$; random-effect model; 9 trials; 405 women in intervention and 389 in placebo group).

In Fang et al.'s meta-analysis in 2016 [2], the mean of fasting blood glucose showed a significantly higher decrease in isoflavone group than placebo group (MD $=-0.22,95 \% \mathrm{CI}=-0.38$ to $-0.07, P=0.004, \mathrm{I}^{2}=79 \%, P<$ 0.00001; random-effect model; 15 trials; 764 in intervention group and 646 in control group).

In Zhang et al's meta-analysis in 2013 [1], a significant borderline decrease was observed in blood glucose in isoflavone supplementation group in comparison with control group $(\mathrm{WMD}=-0.143,95 \% \mathrm{CI}=-0.294$ to $0.009, P=0.065$, heterogeneity $\left[\mathrm{I}^{2}=70 \%\right] ; 12$ trials; 581 
subjects for soy isoflavone supplementation and 601 subjects for control group). However, after exclusion of one study, significant level increased to below 0.05 $(\mathrm{WMD}=-0.189,95 \% \mathrm{CI}=0.344$ to -0.033$)[1]$.

\section{The effect of genistein on the fasting blood glucose}

Three meta-analyses assessed the effect of genistein on the fasting blood glucose. In Fang et al's meta-analysis in 2016 [2], the genistein was more effective than the placebo in attenuating fasting blood glucose (MD $=-0.42 \mathrm{mmol} / \mathrm{L}, P<0.00001,95 \% \mathrm{CI}=-0.54$ to -0.29 $\mathrm{mmol} / \mathrm{L} ; \mathrm{I}^{2}=22 \%, P<0.00001$; random-effect model; 6 trials; 341 for intervention group and 332 for control group). In Liu et al's meta-analysis in 2017 [8], fasting blood glucose in genistein group were significantly lower compared to placebo $(\mathrm{MD}=-6.35 \mathrm{mg} / \mathrm{dL}, P=0.005$, $95 \% \mathrm{CI}=-10.78$ to $-1.93 \mathrm{mg} / \mathrm{dL}$, heterogeneity $\left[\mathrm{I}^{2}=93 \%\right]$, $P<0.00001 ; 6$ trials; 564 women) [8]. In Ricci et al.s meta-analysis [6], fasting blood glucose significantly decreased in the genistein group than the placebo group $(\mathrm{MD}=-7.15 \mathrm{mg} / \mathrm{dL}, P=0.001,95 \% \mathrm{CI}=-11.74$ to $-2.82 \mathrm{mg} / \mathrm{dL}, \mathrm{I}^{2}=94 \%, P<0.00001 ; 3$ trials; 453 women).

\section{HOMA-IR value}

\section{The effect of isoflavones on HOMA-IR value}

In Fang et al's meta-analysis in 2016 [2], the mean HOMA-IR value showed a statistically significant difference in the isoflavones group than placebo group $\left(\mathrm{MD}=-0.52,95 \% \mathrm{CI}=-0.76\right.$ to $0.28, \mathrm{I}^{2}=81 \% ; 12$ trials; random-effect model; 528 women for intervention and 519 for control). In Ricci et al's meta-analysis in 2010 [6], HOMA-IR level showed a larger decrease in isoflavone group compared to placebo $(\mathrm{MD}=-0.39$, $P=0.002,95 \% \mathrm{CI}=-0.65$ to -0.14 , heterogeneity $\left[\mathrm{I}^{2}=74 \%\right]$, $P=0.16 ; 4$ trials; 268 for soy isoflavones and 260 for placebo).

\section{The effect of genistein on HOMA-IR}

Two meta-analyses assessed the effect of genistein on HOMA-IR. Based on the findings of Fang et al's metaanalysis in 2016 [2], the genistein was more effective in comparison to control group in reducing HOMAIR (MD $=-0.66, P<0.00001,95 \% \mathrm{CI}=-0.92$ to -0.41 , $I^{2}=66 \% ; 6$ trials; random-effect model; 6 trials; 341 women for intervention group and 332 placebo group). In Liu et al's meta-analysis in 2017 [8], soy isoflavones were found to be more efficacy than placebo $(\mathrm{MD}=$
$-0.74, P=0.002,95 \% \mathrm{CI}=-1.21$ to -0.28 , heterogeneity $\left[\mathrm{I}^{2}=74 \%\right], P=0.004 ; 5$ trials; 306 for soy gen and 285 for placebo).

\section{DISCUSSION}

The purpose of the current overview was to summarize and critically assess the effect of isoflavones and genistein on glucose metabolism among peri- and post-menopausal women. HOMA-IR value and fasting insulin levels were significantly lower in studies used either isoflavones or genistein compared to placebo. Two meta-analyses showed that treatment with isoflavones could not alter fasting blood glucose [2,6]. However, one of meta-analyses suggested that isoflavones significantly improved blood glucose levels in non-Asian post-menopausal women [8]. We found a discrepancy between mrta anlysis and meta-analyses included to current study. One of the reasons can be attributed to the differences in various methodological and statistical aspects to report findings among the selected trials conducted on non-Asian versus mixed populations reviewed in our meta-analysis.

Several studies have been performed to detect potential mechanisms of the effect of isoflavones on glucose metabolism. For example, the soy in diet can improve the insulin sensitivity due to incremental glucose uptake by skeletal muscles [9]. In-vitro studies showed that several mechanisms of action may involve in the effect of soy on the glucose metabolism, such as an inhibitory action of tyrosine kinase, changes in the number of insulin receptor, impaired glucose transport and intracellular phosphorylation [6]. Villa et al. [10] divided 54 postmenopausal women into two groups of receiving $54 \mathrm{mg} /$ day of genistein and placebo. Genistein affected significantly the glycoinsulinemic metabolism. They also performed a subgroup analysis in the patients of genistein group based on hyperinsulinemic and normoinsulinemic subjects. Their results revealed that glycemic indexes were improved in hyperinsulinemic group [10].

The current overview has several limitations that should be discussed. There were moderate to high heterogeneities in all reviews included to our overview. Based on AMSTAR criteria, at least two databases must be searched, and graphical plot should be drown in combination with statistical test when reporting publication bias. One of the studied reviews only searched one database and some of meta-analyses evaluated 
neither Begg's nor Egger's tests in combination with graphical plot. Hence, the publication bias might affect their studies. List of included and excluded studies were not reported in two out of four reviews. Moreover, none of authors of systematic reviews stated whether they include grey literature. Future systematic review and meta-analyses should be well designed to meet AMSTAR criteria.

\section{CONCLUSION}

Regardless of population, the treatment with genistein is effective on improving fasting insulin, HOMA-IR and glucose levels. Nevertheless, the high heterogeneity among studies and poor methodology of reviews make it difficult to draw a definite conclusion on the positive impacts of soy on glucose metabolism.

\section{CONFLICT OF INTEREST}

No potential conflict of interest relevant to this article was reported.

\section{REFERENCES}

1. Zhang YB, Chen WH, Guo JJ, Fu ZH, Yi C, Zhang M, et al. Soy isoflavone supplementation could reduce body weight and improve glucose metabolism in non-Asian postmenopausal women: a meta-analysis. Nutrition 2013; 29: 8-14.

2. Fang K, Dong H, Wang D, Gong J, Huang W, Lu F. Soy isoflavones and glucose metabolism in menopausal women: a systematic re- view and meta-analysis of randomized controlled trials. Mol Nutr Food Res 2016; 60: 1602-14.

3. Sumino H, Ichikawa S, Yoshida A, Murakami M, Kanda T, Mizunuma $\mathrm{H}$, et al. Effects of hormone replacement therapy on weight, abdominal fat distribution, and lipid levels in Japanese postmenopausal women. Int J Obes Relat Metab Disord 2003; 27 : 1044-51.

4. Ishimi Y. Soybean isoflavones in bone health. In: Yoshikawa HT, editor. Food factors for health promotion. Basel: Karger Publishers; 2009. pp. 104-16.

5. Cheng SY, Shaw NS, Tsai KS, Chen CY. The hypoglycemic effects of soy isoflavones on postmenopausal women. J Womens Health (Larchmt) 2004; 13: 1080-6.

6. Ricci E, Cipriani S, Chiaffarino F, Malvezzi M, Parazzini F. Effects of soy isoflavones and genistein on glucose metabolism in perimenopausal and postmenopausal non-Asian women: a metaanalysis of randomized controlled trials. Menopause 2010; 17: 1080-6.

7. Oxman AD, Schünemann HJ, Fretheim A. Improving the use of research evidence in guideline development: 8 . Synthesis and presentation of evidence. Health Res Policy Syst 2006; 4: 20.

8. Liu Y, Li J, Wang T, Wang Y, Zhao L, Fang Y. The effect of genistein on glucose control and insulin sensitivity in postmenopausal women: a meta-analysis. Maturitas 2017; 97: 44-52.

9. Cederroth CR, Nef S. Soy, phytoestrogens and metabolism: a review. Mol Cell Endocrinol 2009; 304: 30-42.

10. Villa P, Costantini B, Suriano R, Perri C, Macrì F, Ricciardi L, et al. The differential effect of the phytoestrogen genistein on cardiovascular risk factors in postmenopausal women: relationship with the metabolic status. J Clin Endocrinol Metab 2009; 94: 552-8. 